\title{
Evolution of alternative male morphotypes in oxyurid nematodes: a case of convergence?
}

\author{
F. JORGE* †+, A. PERERA*, V. ROCA§, M. A. CARRETERO*, D. J. HARRIS* \& R. POULIN \\ *CIBIO Research Centre in Biodiversity and Genetic Resources, InBIO, Universidade do Porto, Vairão, Portugal \\ $\dagger$ Departamento de Biologia, Faculdade de Ciências da Universidade do Porto, Porto, Portugal \\ $\$$ Department of Zoology, University of Otago, Dunedin, New Zealand \\ $\S$ Departament de Zoologia, Facultat de Ciències Biològiques, Universitat de València, València, Spain
}

Keywords:

alternative adaptation;

alternative reproductive tactics;

male dimorphism;

parasites;

polymorphism.

\begin{abstract}
Male dimorphism has been reported across different taxa and is usually expressed as the coexistence of a larger morph with exaggerated male traits and a smaller one with reduced traits. The evolution and maintenance of male dimorphism are still poorly understood for several of the species in which it has been observed. Here, we analyse male dimorphism in several species of reptile parasitic nematodes of the genus Spauligodon, in which a major male morph (exaggerated morph), which presents the traditional male morphological traits reported for this taxon, coexists with a minor morph with reduced morphological traits (i.e. reduced genital papillae) resembling more closely the males of the sister genus Skrjabinodon than Spauligodon major males. Because of the level of uncertainty in the results of ancestral state reconstruction, it is unclear if the existence of male dimorphism in this group represents independent instances of convergent evolution or an ancestral trait lost multiple times. Also, although the number of major males per host was positively correlated with the number of females, the same did not hold true for minor males, whose presence was not associated with any other ecological factor. Nevertheless, the existence of male dimorphism in Spauligodon nematodes is tentatively interpreted as resulting from alternative reproductive tactics, with differences in presence and number of individuals as indicators of differences in fitness, with the lower numbers of minor males per host likely maintained by negative frequency-dependent selection.
\end{abstract}

\section{Introduction}

Deviating from the general trend promoting a single morphology in each sex, there are several taxa where, rather than giving rise to a single fittest male and/or female phenotype, evolution has instead resulted in extreme phenotypic diversity (multiple adaptive peaks) with the existence of multiple morphs in the two sexes (Gross, 1996). Alternative male phenotypes have been reported for a variety of species across different taxa,

Correspondence: Fátima Jorge, CIBIO, Research Center in Biodiversity and Genetic Resources, Campus Agrário de Vairão, Rua Padre Armando Quintas, Vairão 4485-661, Portugal.

Tel.: +351 252660411 ; fax: +351 252661780 ;

e-mail: fatima.esperanca@cibio.up.pt from invertebrates (i.e. arthropods: Buzatto et al., 2011; nematodes: Hoberg et al., 2012) to vertebrates (i.e. birds: Horton et al., 2012; fishes: Cogliati et al., 2013; reptiles: Calsbeek et al., 2010). The existence of alternative male phenotypes may result from alternative adaptation, in the broad sense (West-Eberhard, 1986), or alternative reproductive tactics (ART), that is, conspecific, intrasexual competitors (Gross, 1996; Tomkins \& Hazel, 2007; Oliveira et al., 2008). However, in both cases the underlying mechanisms that regulate phenotypic alternatives may be similar, and selection against intermediate phenotypes may lead to subsequent establishment of distinct intraspecific alternative forms (West-Eberhard, 1986; Taborsky et al., 2008). In the first case, different adaptive phenotypes maintained in the same life stage and the same population may result from epigenetic divergence (i.e. heterochrony), giving 
rise to what West-Eberhard (1986) called intraspecific 'alternative adaptations'. This allows a single species to occupy more than one sympatric niche, thus increasing its adaptive potential (natural selection). Species with alternative strategies may also be less vulnerable to extinction and better able to adapt to new environments than monomorphic species (Pizzatto \& Dubey, 2012; Bastiaans et al., 2013). In the second case, evolution of male phenotypes might represent different solutions to reproductive competition (sexual selection), rather than a broader adaptive potential. ART are expected to arise whenever there is increased fitness to be gained by pursuing different reproductive tactics consisting in the specialization of same-sex conspecifics to exploit different reproductive niches, with their frequencies depending on the reproductive potential of each niche (Taborsky et al., 2008). Several theoretical frameworks around the strategies involved in ART have been proposed and the maintenance of alternative phenotypes has been widely discussed (see Gross, 1996; Shuster \& Wade, 2003; Oliveira et al., 2008). However, most cases of ART probably result from both genes and environment contributing to the phenotypic expression of the different tactics (Brockmann, 2001; Neff $\delta$ Svensson, 2013). The coexistence of multiple morphs, in the context of ART, is believed to be maintained through negative frequency-dependent selection (Iserbyt et al., 2013). Such alternative phenotypes are expected to persist in a population when their fitness curves cross, that is, when each does better than the other under some conditions (Brockmann, 2001).

One of the most striking observations in male dimorphism is the convergence to similar general phenotypes shared across unrelated taxa usually involving allometric differences between several morphological traits, with a smaller male phenotype (or reduced morph, MI) and a larger one (or exaggerated morph, MA). Why are particular solutions so similar and frequent across a wide range of taxa? In the case of ART, this convergent selection may reflect a similar underlying process shaping its evolution across independent lineages (Mank \& Avise, 2006).

Given that in many cases taxonomy is still mainly based on morphological characters, the assessment of male dimorphism is of relevance in species description and has potential implications for taxonomy and systematics. This is especially true in groups such as parasitic nematodes, where genus diagnosis is frequently based on male morphology (i.e. Nematoda, Oxyurida: Ainsworth, 1990; Rhabditida: Hoberg et al., 2012). For example, in oxyurid nematodes, Ainsworth (1990) identified male dimorphism in two species of Skrjabinodon nematodes, with morphological differences between male morphs consistent between the two species. Male dimorphism is also quite common in Ostertagiinae nematodes (Hoberg \& Abrams, 2001; Grillo et al., 2008; Hoberg et al., 2012). In one representative of this group, Teladorsagia circumcincta, the frequency of morphological polymorphism is primarily density dependent, with minor male morphs more likely to occur in high intensity infections, their intensity being positively correlated with that of the larger morph (Craig et al., 2010). However, differences in mating tactics of the different male morphotypes are still unknown among nematodes.

In this study, we combine genetic and ecological data to investigate the presence of male dimorphism across several species of the oxyurid nematode genus Spauligodon that infect reptiles. Specifically, we test the hypotheses that (i) similar patterns of male dimorphism have evolved convergently within different species and (ii) that the occurrence of the minor male morphs is frequency-dependent in natural infections as a possible consequence of ART. To test these two hypotheses, we build a phylogenetic framework to map the occurrence of dimorphic male morphotypes among Spauligodon species and statistically assess the role of ecological factors in driving the presence and extent of male dimorphism.

\section{Materials and methods}

\section{Study system}

Nematodes of the order Oxyurida are haplodiploid, that is, males derive from unfertilized eggs and are haploid, whereas females are diploid and develop from fertilized eggs (Adamson, 1990). Within the order, members of the family Pharyngodonidae Travassos, 1919 are usually identified on the basis of male diagnostic morphological characters, females generally being very similar between groups. The main feature used to separate Spauligodon Skrjabin, Schikhobalova and Lagodovskaja, 1960 from other genera of the Pharyngodonidae family is primarily based on male morphology, namely the presence of caudal alae not supported by the last pair of genital papillae. The closely related genus, Skrjabinodon Inglis, 1968, is morphologically similar but lacks caudal alae, and their genital papillae are sessile and often reduced. Within the genus Spauligodon, species identification also relies on other male features, that is, shape of the caudal papillae and genital cone. Females are often indistinguishable between the two genera. In the Mediterranean and Macaronesian regions, the two genera are usually found in sympatry, infecting the same reptile host species (Roca et al., 1989, 2005; Hornero \& Roca, 1992).

\section{Sampling procedures}

A total of 916 samples, consisting of 593 intestines and 323 faecal pellets, were collected from 12 lizard species from different localities between 2009 and 2012 (Table 1; Table S1, Supporting information). Faecal samples were obtained either through spontaneous defecation of the reptiles when captured, or by gentle 
abdominal massage. Intestines were removed from dead animals, which accidentally died during fieldwork or that were euthanized through inhalation of ether vapours. All samples were preserved in 96\% ethanol and examined for helminths, which were then separated, counted and identified. Spauligodon spp. specimens were mounted on temporary slides with a glycerol : water (1:1) solution and observed at different magnifications using a light microscope (Olympus CX41, Olympus Australia Pty Ltd, Notting Hill Victoria, Australia). Representatives of each male morph from each species were genetically characterized. Prior to extraction specimens were photographed using a digital camera Olympus DP25 (Olympus ${ }^{\circledR}$, Tokyo, Japan) and measured with the DP2-BSw software (Olympus ${ }^{\circledR}$ ).

\section{Male morph discrimination}

In all cases, two male morphs could be unambiguously identified based on several morphological features (Table 2, Fig. 1). The major male morph (MA) corresponded to the phenotype typically associated with Spauligodon males, that is, presence of a caudal alae not supported by the last pair of genital papillae, genital cone present and genital papillae well developed and often pedunculated (Fig. Ib,c). The minor male morph (MI) corresponded to males displaying the Skrjabinodon typical morphological features, that is, caudal alae and genital cone absent, genital papillae sessile and reduced, curled posterior end and spicule present (Table 2, Fig. le,f). The MI was preliminary assigned to a particular Spauligodon species according to the respective conspecific MA males found in the same host individual, or within the same host population. In all cases, this assignation was later confirmed by genetic analyses.

\section{DNA extraction and sequencing}

Extraction of genomic DNA was performed on individual nematodes using the PureLink ${ }^{\circledR}$ Genomic DNA Kit (Invitrogen, Invitrogen New Zealand Ltd, Auckland, New Zealand) according to the manufacturer's protocol. To avoid cross-contamination, the two types of male morphs were extracted separately. Additional specimens from several Spauligodon species were also extracted (Table 3) to provide a more robust phylogenetic inference. Three partial gene fragments were amplified: two nuclear genes, the $18 \mathrm{~S}$ ribosomal RNA (18S) and $28 \mathrm{~S}$ ribosomal RNA (28S) and one mitochondrial gene, the cytochrome oxidase subunit I (COI). The $18 \mathrm{~S}$ was amplified using the primers Nem $18 \mathrm{~S}$ F and Nem $18 \mathrm{~S} R$ from Floyd et al. (2005). For the amplification of the $28 \mathrm{~S}$ fragment, primers $28 \mathrm{~S} \mathrm{rDl} .2 \mathrm{a}$ and $28 \mathrm{~S}$ B described by Whiting (2002) were used. The COI fragment was amplified using the nematode cocktail primers C_NemFl_tl and C_NemRl_tl from Prosser et al. (2013). Polymerase chain reactions (PCR) were performed in a total volume of $20 \mu \mathrm{L}$, comprising $4 \mu \mathrm{L}$ of MyTaq $^{\text {TM }}$ Red reaction buffer (Bioline, Bioline (Aust) Pty Ltd, Alexandria, NS W, Australia), primers at $0.5 \mathrm{~mm}$ each, $0.1 \mu \mathrm{L}$ of $\mathrm{MyTaq}^{\mathrm{TM}}$ Red DNA Polymerase (Bioline) and 2-3 $\mu \mathrm{L}$ of extracted nematode DNA template. For all the sets of primers, PCR consisted of 35 iterations of the following cycle: $40 \mathrm{~s}$ at $95{ }^{\circ} \mathrm{C}, 40 \mathrm{~s}$ at $45-54{ }^{\circ} \mathrm{C}$ (depending on the primers used) and $1 \mathrm{~min}$ at $72{ }^{\circ} \mathrm{C}$, beginning with an additional denaturation step of 3 min at $95^{\circ} \mathrm{C}$ and ending with a final extension at $72{ }^{\circ} \mathrm{C}$ for $10 \mathrm{~min}$. Amplified $18 \mathrm{~S}$ and $28 \mathrm{~S}$ fragments were sequenced for both strands with the same primers used in the amplification process, whereas for COI, following Prosser et al. (2013), the primers M13F

Table 1 Prevalence (\%), mean intensity (\%), range and total number of individuals recovered of the two male morphotypes of each Spauligodon species, found in each type of sample, faeces and intestines.

\begin{tabular}{|c|c|c|c|c|c|c|c|c|c|c|}
\hline Species & N Host & P MA & $\mathrm{PMl}$ & I MA & MA range & I Ml & MI Range & N MA & N Ml & MA/MI \\
\hline \multicolumn{11}{|l|}{ Intestines } \\
\hline S. occidentalis & 101 & 27.72 & 3.96 & 14.96 & 1 to 88 & 1 & - & 419 & 4 & 104.80 \\
\hline Spauligodon sp. PS* & 2 & 0.00 & 50.00 & 0.00 & - & 1 & - & 0 & 1 & 0.00 \\
\hline S. carbonelli & 20 & 70.00 & 5.00 & 7.71 & 1 to 21 & 1 & - & 108 & 1 & 108.00 \\
\hline S. saxicolae & 397 & 2.27 & 4.28 & 1.44 & 1 to 3 & 1 & - & 13 & 17 & 0.76 \\
\hline \multicolumn{11}{|l|}{ Faeces } \\
\hline S. occidentalis & 106 & 26.42 & 7.55 & 6.04 & 1 to 30 & 1 & - & 169 & 8 & 21.13 \\
\hline S. atlanticus & 105 & 21.90 & 3.81 & 5.91 & 1 to 27 & 1 & - & 136 & 4 & 34.00 \\
\hline Spauligodon sp. TG† & 10 & 20.00 & 20.00 & 11.00 & 5 to 17 & 2 & 1 to 3 & 22 & 4 & 5.50 \\
\hline Spauligodon sp. PS* & 83 & 7.23 & 1.59 & 2.67 & 1 to 9 & 1 & - & 16 & 1 & 16.00 \\
\hline S. carbonelli & 19 & 0 & 0 & 0 & - & 0 & - & 0 & 0 & - \\
\hline
\end{tabular}

MA, major male morphotype; MI, minor male morphotype; N Host, number of sampled hosts; P, Prevalence; I, Intensity; N MA, total number of MA; N MI, total number of MI; MA/MI, male morphs ratio (total number of MA/total number of MI).

* Spauligodon sp. lineage infecting Podarcis tiliguerta.

$\uparrow$ Spauligodon sp. lineage infecting Tarentola gigas. 


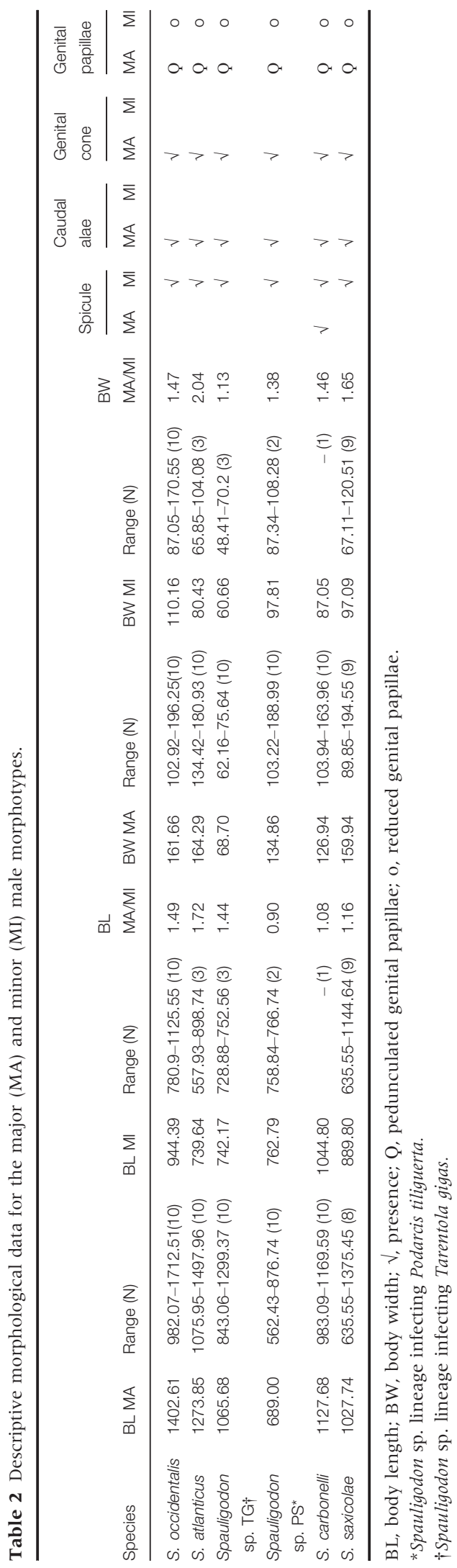

and Ml3R (Messing, 1993) were used. PCR product purification and sequencing was performed by a commercial facility (Macrogen Corporation, http:// www.macrogen.com).

\section{Phylogenetic analysis}

The obtained sequences were imported into the software GeneIous 6.1.2 (Biomatters, 2013) where contiguous sequences were assembled. Additional Spauligodon sequences published in previous studies were also included (Table 3). Parapharyngodon echinatus and Thelandros tinerfensis were used as outgroups for the $18 \mathrm{~S}$ and 28S data sets, whereas Spauligodon anolis was used as an outgroup for the COI data set. Sequences where aligned with MAFFT 7.017 (Katoh et al., 2002) implemented in GENEIOUS 6.1.2 using the default parameters (auto algorithm; scoring matrix $=200 \mathrm{PAM} / \mathrm{k}=2$; gap open penalty $=1.53$; and offset value $=0.123$ ), followed by minor manual editing. For the COI, a total of 641 unambiguously aligned positions were obtained. The $18 \mathrm{~S}$ and $28 \mathrm{~S}$ alignments resulted in a total of 858 positions and 1133 positions including gaps, respectively. To determine the best fitting nucleotide model for the data set, the software JMODELTEST 2 (Darriba et al., 2012) was used under the Akaike Information Criterion (AIC). The models selected were: $\mathrm{GTR}+\mathrm{I}, \mathrm{GTR}+\mathrm{I}+\mathrm{G}$ and $\mathrm{TIM} 3+\mathrm{I}+\mathrm{G}$ for the $18 \mathrm{~S}, 28 \mathrm{~S}$ and COI data sets, respectively. Phylogenetic analyses were performed using Bayesian inference (BI) and maximum likelihood (ML) methods, implementing the most appropriate parameters according to the estimated models. Bayesian analyses were performed in MRBAYES 3.2.1 (Huelsenbeck \& Ronquist, 2001) and ran for $10 \times 10^{6}$ generations with random starting trees, sampling every 100 generations. The first 25000 trees were discarded as 'burn-in', after verifying that stationarity was reached by plotting log-likelihood values against generation time. A 50\% majority-rule consensus tree was used to summarize the trees sampled from the post-burn-in trees. Maximum likelihood analyses were performed using PHYML 3.0 (Guindon \& Gascuel, 2003). Branch support was estimated by bootstrap analysis (Felsenstein, 1985) with 1000 replicates. Additionally, to obtain a more robust inferred phylogeny to use in the ancestral state reconstruction (ASR), a BI was also performed for the combined data set of $28 \mathrm{~S}$ and COI including only a single representative for each species, using P. echinatus and T. tinerfensis as outgroups. Data were partitioned by gene, implementing the most appropriate parameters according to the respective estimated model for each partition. BI of the combined data was performed as described above. Estimates of pairwise uncorrected differences (p-distance) were made in MEGA 5 (Tamura et al., 2011). New sequences generated in this study were submitted to GenBank (Table 3). 


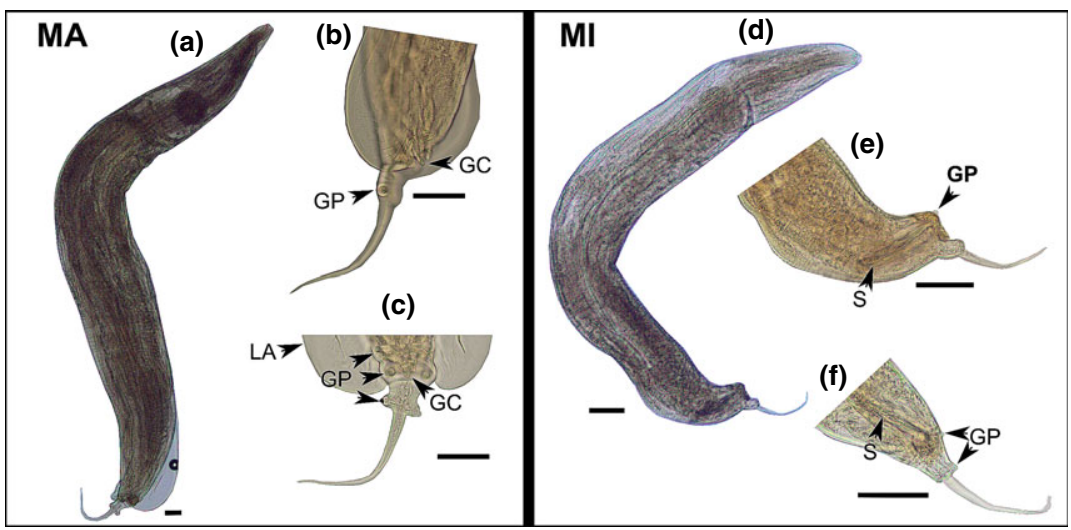

Fig. 1 Spauligodon major $(\mathrm{a}-\mathrm{c})$ and minor $(\mathrm{d}-\mathrm{f})$ morphotypes. (a), general view of the major morph; (b), lateral view of major morph caudal extremity; (c), ventral view of major morph caudal extremity; (d), general view of the minor morph; (e); lateral view of minor morph caudal extremity; (f), ventral view of minor morph caudal extremity; MI, minor morph; MA, major morph. GP, genital papillae, GC, genital cone; S, spicule; LA, lateral alae. Scale $50 \mu \mathrm{m}$.

\section{Analysis of dimorphism evolution}

The occurrence of male dimorphism was mapped on the inferred phylogeny based on the combined $28 \mathrm{~S}$ and COI data, as a binary categorical trait: absent $(0)$ or present (1). The evolution of male dimorphism was reconstructed under parsimony (MP) and likelihood (ML) based ancestral state reconstruction (ASR) approaches. Although MP minimizes the number of character state changes, ML methods consider every possible reconstruction, estimating the ancestral states that maximize the probability of the observed states evolving under a stochastic model (Cunningham et al., 1998). ASRs were performed using the ancestral state module implemented in Mesquite v.2.75 (Maddison \& Maddison, 2011) using the option 'trace character history'. The ancestral states were summarized on the $50 \%$ majority-rule consensus $28 \mathrm{~S}$ and COI BI tree. We used the Markov k-state 1 (Mkl) model of evolution for the ML reconstructions, which assumes a single rate of transition between two character states, and in which any particular change is equally probable between the two-state character (presence or absence of male dimorphism). This model was implemented with the default settings (threshold when decisions made: 2.0). To facilitate comparison between MP and ML methods, both reconstructions were visualized under the 'Balls \& Sticks' tree form. Although pies at the nodes represent relative likelihoods for ML reconstruction, in MP reconstruction pies only represent the estimated ancestral state.

\section{Factors related to $\mathrm{MI}$ and MA presence and abundance}

Our data set included the following variables: parasite species, host species, type of sample (intestines or faeces), number of hosts infected with the MA male morph, number of hosts infected with the MI male morph, number of MA male morphs per host, number of MI male morphs per host, number of females per host, sum of the two male morphs per host, operational sex ratio [total number of adult males/(total number of adult males + total number of adult females), following Kvarnemo \& Ahnesjö, 1996], total number of Spauligodon specimens per host, total number of other helminth species per host and total number of helminths per host.

Parasitism was expressed at the host species level by calculating prevalence and mean intensity. Parasite prevalence was calculated as the ratio between the number of infected host individuals and the total number of sampled host individuals, and parasite mean intensity as the mean number of parasites per infected host. Given the differences in detectability and abundance of nematodes depending on the origin of the samples (see Jorge et al., 2013), data retrieved from intestines and faecal samples were treated separately in further analyses. A Wilcoxon sign-rank test with continuity correction (function wilcoxon.test of the $\mathrm{R}$ package) was performed to determine if there were differences in the presence and number of MI and MA per host, across all hosts. We further tested any correlation between number of MA morph and other variables using a nonparametric Spearman correlation (function rcorr, R package Hmisc, Harrell, 2013). This test was not performed for the variable 'number of MI male morphs per host', as all host specimens analysed, with the exception of one, were infected with a single MI individual.

Firstly, to test whether the presence of MI could be determined by the number of MA male morphs, we performed a generalized linear model with a logistic regression function using a binomial distribution with 


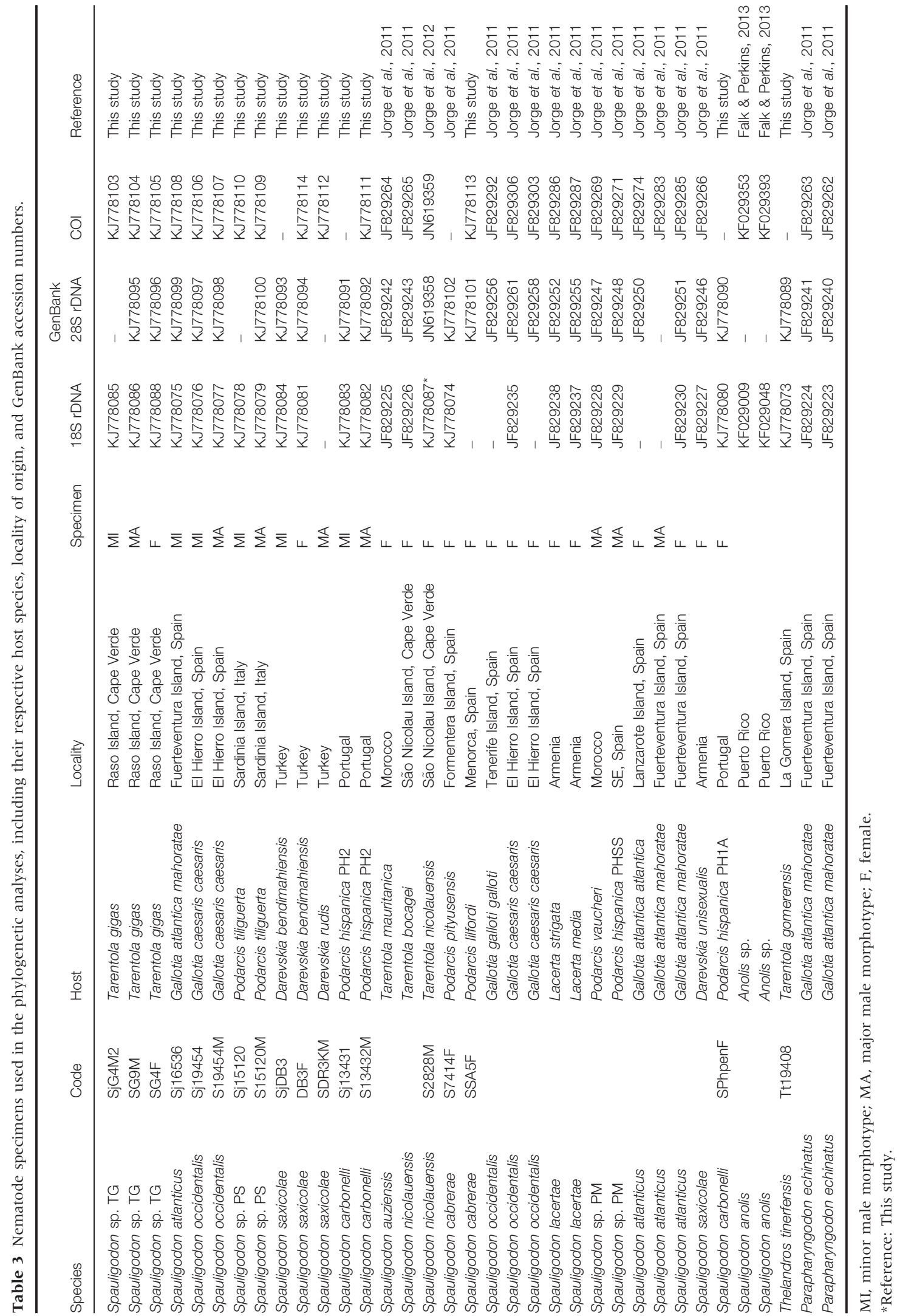


the presence or absence of MI morph as response variable and the number of MA as the predictor. Due to the low number of individuals infected in some host species (see Table 1), analyses were performed in pooled host species. Secondly, to test the hypothesis that MI represents an alternative male strategy, we constructed a generalized linear mixed model fitting the presence of MI morph as response variable and operational sex ratio, number of females, number of other helminths and total number of helminths and the interaction between operational sex ratio and number of females as predictors. We controlled for variation between different host species by also including host identity as a random factor. The operational sex ratio is a good predictor of male contest competition for mates (Kvarnemo \& Ahnesjö, 1996; Shuster \& Wade, 2003). The total number of females and its interaction with operational sex ratio were included in the model to account for the possibility that the availability of females is more important than male competition. The number of other helminths and the total number of helminths were included as main factors to assess whether the presence of MI morph was related to the presence and number of other helminths. No other variables were included in the model to prevent over parameterization (see Burnham \& Anderson, 2002). All variables were standardized prior to analyses (standardize function, R package arm, Gelman et al., 2009). A full submodel set (including the null model) from the global model was created using the dredge function implemented in the MuMIn package (Bartoń, 2009). Even considering the small size of our data set, we decided to employ information theoretic model averaging approach, a procedure that accounts for model selection uncertainty to obtain robust parameter estimates or predictions (Grueber et al., 2011). Model averaging was performed on the best submodels $(\triangle \mathrm{AICc}<4)$, obtained with the function get.models and after using the model.avg function from MuMIn R package (Barton, 2009). As we aimed to determine which factors had the strongest effect on the response variable, we used the zero method for model averaging (Nakagawa \& Freckleton, 2010). To determine if the results obtained in our analyses were affected by the nature of the response variable (low presence of MI male morph) resulting in a large amount of absences, a post hoc resampling procedure was performed. Given the low reliability of the faecal samples for capturing the helminth communities in the intestine (Jorge et al., 2013), the analysis was conducted only for intestine samples. In each resampling, a sub-data set was generated consisting of the 24 samples where MI male morphs were found and an additional 24 random samples where this morph was absent. For each sub-data set, we used the model averaged from the best submodels $(\Delta \mathrm{AICc}<4)$ obtained in the previous analysis (i.e. a generalized linear mixed model fitting the presence of MI morph as response variable and operational sex ratio, number of females, number of other helminths and total number of helminths as predictors), implementing it as our new global model. We then performed a similar analysis as described above, with the exception that the new model average was performed over all the submodels. This procedure was repeated 1000 times with resampling without replacement over the absences data. All analyses were implemented using the package $\mathrm{R}$ version 3.0.2 (R Core Team, 2013).

\section{Results}

\section{Phylogenetic analyses}

A total of $858 \mathrm{bp}$ was used in the $18 \mathrm{~S}$ analyses, whereas for the 28S and COI $1133 \mathrm{bp}$ and $641 \mathrm{bp}$ were used, respectively. For the combined COI and 28S data, 1774 bp was assembled. The 18S was the least informative marker with only 21 variable sites (29 including Spauligodon anolis sequences), against 154 for the $28 \mathrm{~S}$ and 250 in COI (in all cases excluding respective outgroups). Nevertheless, all three markers retrieved the same pattern, with the MA and MI morphotypes clustering together irrespective of the species assigned (Figs 2 and 3), despite the unresolved topology between some branches. Estimates of sequence divergence between each pair of male morphotypes were never higher than $0 \%$ for the $18 \mathrm{~S}, 0.1 \%$ for $28 \mathrm{~S}$ or $0.8 \%$ for COI. We were not able to successfully amplify both $28 \mathrm{~S}$ and COI markers for the two male morphotypes of Spauligodon saxicolae. However, the only MI amplified (SjDB3) clusters within the other specimens of $S$. saxicolae from Turkey, confirming its identification as a male morphotype of that species (Fig. 3).

\section{Analysis of dimorphism evolution}

The results from the ASR of male dimorphism in Spauligodon nematodes mapped over the $50 \%$ majority-rule consensus BI tree are represented in Fig. 4. When ASR was performed under a parsimony approach, presence of MI was retrieved as the ancestral state, whereas in the likelihood approach the reconstruction yielded 50\% probability for each alternative state (ancestral vs. derived).

\section{Factors related to $\mathrm{MI}$ and MA presence and abundance}

Prevalence and mean intensities of the two male morphs are presented in Table 1. The analysis of prevalence and intensity showed that the MA was significantly more prevalent than the MI [Wilcoxon sign-rank test with continuity correction; all pooled species: $\mathrm{W}=1347.5, \quad P<0.005$ in faecal samples $(n=64)$; $\mathrm{W}=2223, P<0.005$ in intestine samples $(n=81)]$ and 


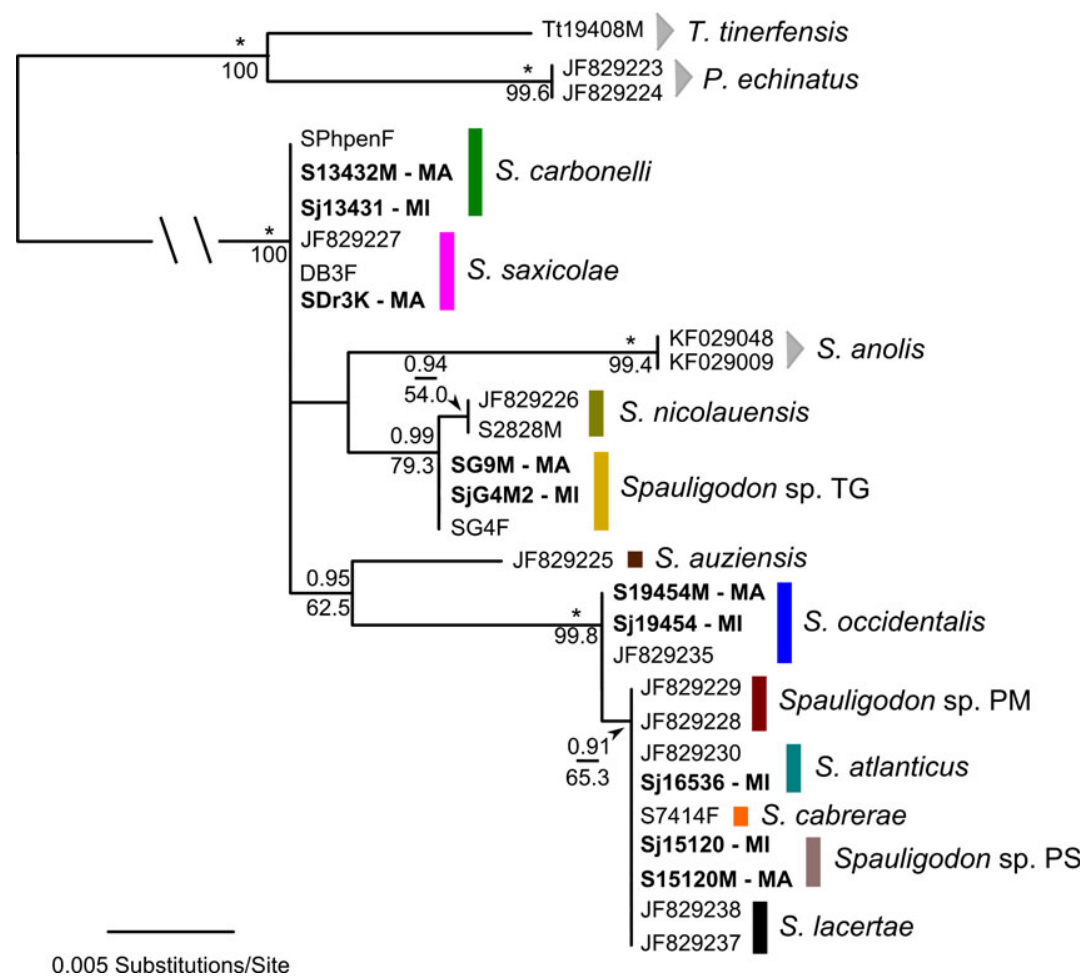

Fig. 2 Maximum likelihood inference tree derived from 18S rRNA gene sequences. Values above branches represent Bayesian posterior probabilities and those below represent ML bootstrap support values (posterior probabilities below 0.75 and bootstrap values below 50 are not reported). *Corresponds to a posterior probability value of 1 . Male morph specimens are in bold. For species details see Table 3.

attained higher intensities (all pooled species: $\mathrm{W}=1963.5, P<0.005$ in faecal samples; $\mathrm{W}=2921$, $P<0.005$ in intestine samples). Although the number of MA per host ranged from 1 to 88 individuals, only one MI per host was found, with the exception of a single host that harboured three MI individuals (Table 1). The number of MA per host was positively correlated with the number of females (faeces: rho $=0.63, \quad P<0.001 ; \quad$ intestines: $\quad$ rho $=0.55$, $P<0.001$ ) and the total number of helminths (faeces: rho $=0.4, \quad P=0.0012$; intestines: rho $=0.78$, $P<0.001)$, in both types of samples. Similarly, the total number of males was also positively correlated with number of females (faeces: rho $=0.6$, $P<0.001$; intestines: rho $=0.52, \quad P<0.001)$ and total number of helminths (faeces: rho $=0.42$, $P<0.001$; intestines: rho $=0.76, P=0)$. Regarding the relationship between the two male morphs, there was a significant association between the intensity of MA and the presence of MI in faeces, but not in intestine samples (Faeces: $F_{1,62}=7.021$, $P=0.012$; intestines: $\left.F_{1,79}=1.407, P=0.264\right)$.

The cut-off of 4AICc yielded five and seven models for the presence of the MI morph among faecal and intestinal samples, respectively, which were then included in the model averaging. For data collected from faeces, all parameters were included in the model averaging, but not for data collected from intestines, for which there was no interaction between number of females and operational sex ratio. The results from the model averaging after standardization (effect sizes are therefore on a comparable scale) are summarized in Table 4. From the faeces data, operational sex ratio and number of females were the most important predictors ( $100 \%$ relative importance) of MI presence, together with their interaction $(84 \%)$. However, only the confidence intervals of OSR do not include zero and therefore only this factor is significant at $\alpha=0.05$. For the intestine data, no parameter was found to have an importance higher than $30 \%$ and all of their respective confidence intervals included zero. In the post hoc analysis, of the 1000 repetitions performed, we found significant results in only $12.3 \%$ of cases for the number of females per host, $6.9 \%$ for the total number of helminths per host, $1.4 \%$ for the operational sex ratio and $1.0 \%$ for the total number of other helminth species per host. The average relative importance of each of these predictors was never higher than $40 \%$ (total number of other helminth species per host: $40.99 \% \pm 11.95$; operational sex ratio: $32.98 \% \pm 5.59$; 
(a)
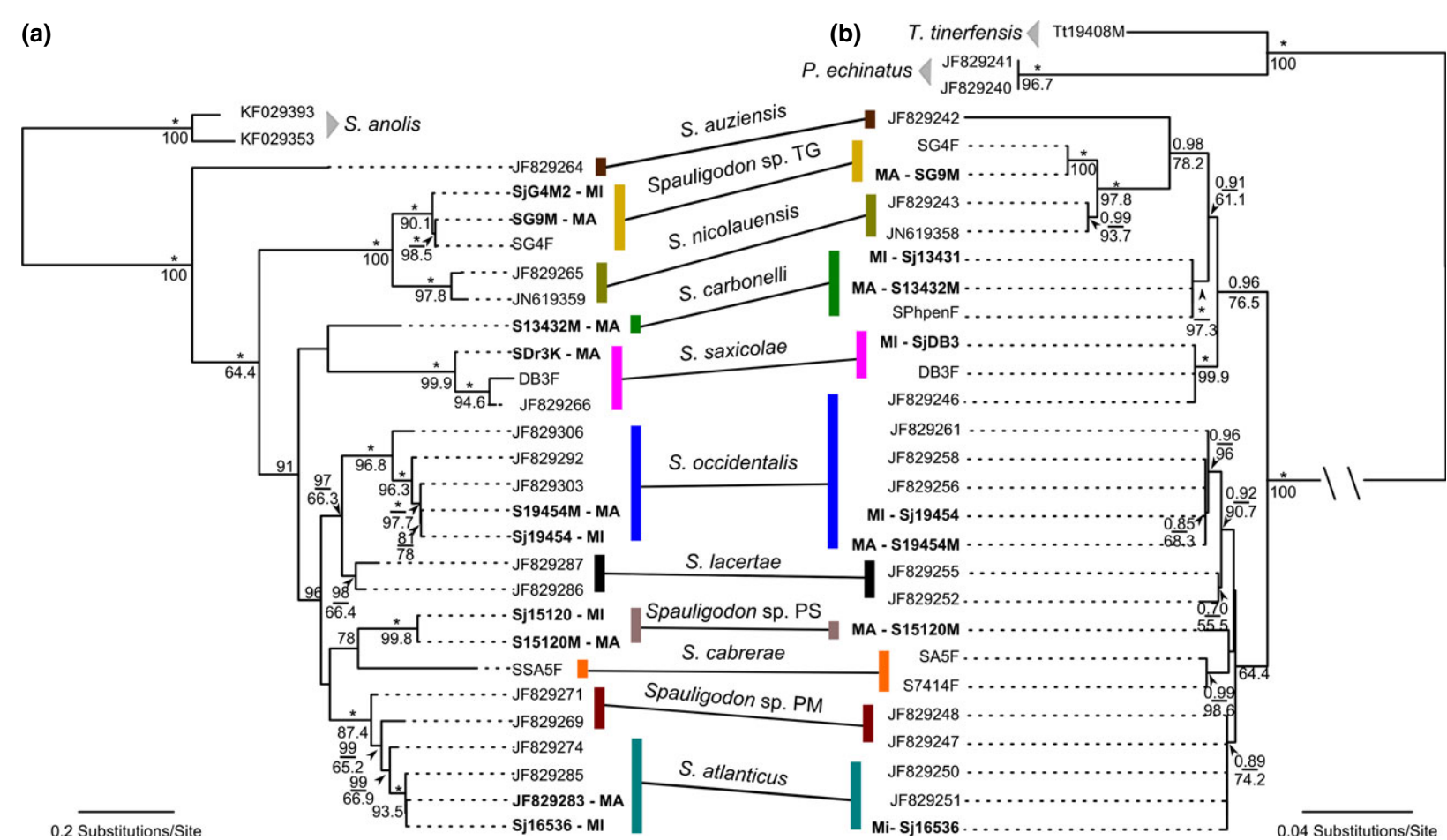

Fig. 3 Maximum likelihood inference trees derived from cytochrome oxidase subunit I (a) and 28S rRNA (b) gene sequences. Values above branches represent Bayesian posterior probabilities and those below represent ML bootstrap support values (posterior probabilities below 0.75 and bootstrap values below 50 are not reported). ${ }^{*}$ Corresponds to a posterior probability value of 1. Male morph specimens are in bold. For species details see Table 3.

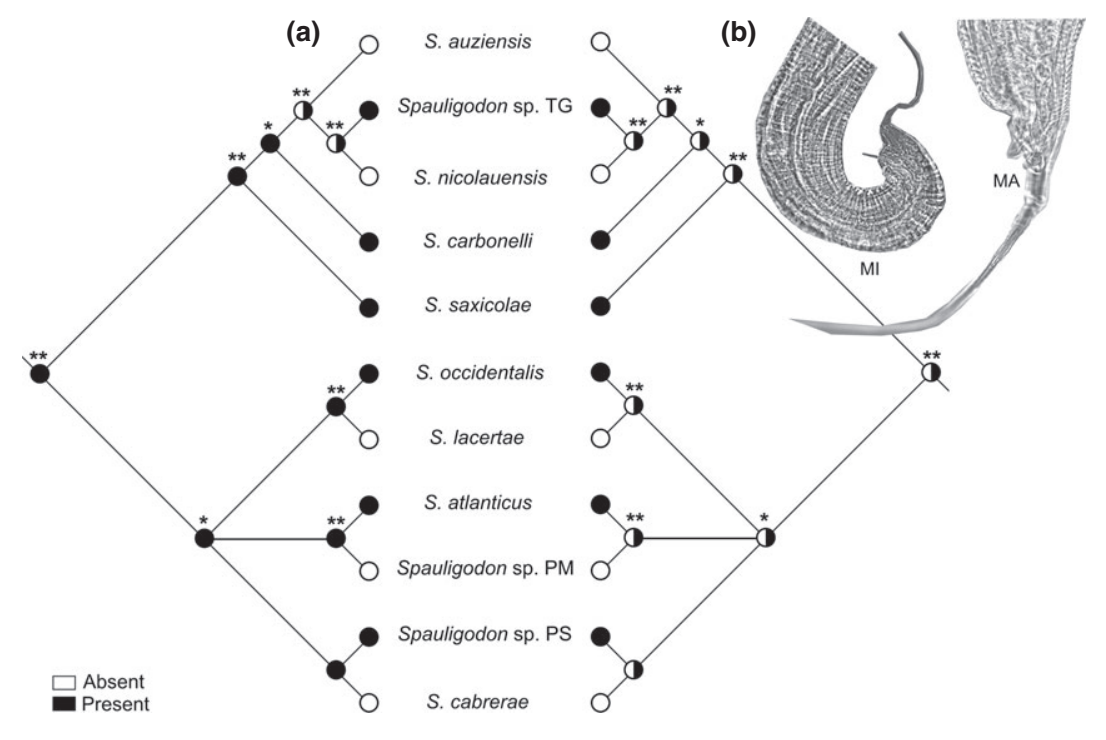

Fig. 4 Ancestral state reconstructions for the Spauligodon male dimorphism based on parsimony (a) and likelihood (b), traced under $50 \%$ majority-rule consensus $28 \mathrm{~S}$ and cytochrome oxidase subunit I Bayesian inference tree. Pie diagrams at each node: (a) parsimony reconstruction indicative of estimated ancestral state, (b) reconstruction indicate relative likelihoods for ML reconstruction; Asterisks denote Bayesian posterior probabilities values: *, 78-90\%;**, 91-100\%. For species details see Table 3. 
Table 4 Summary results of the effects of several factors on the presence of the MI morph after model averaging. Analyses were performed separately for intestinal and faecal samples.

\begin{tabular}{|c|c|c|c|c|c|}
\hline & & Estimate $^{\star}$ & Standard error & Confidence interval & Relative importance \\
\hline \multirow[t]{6}{*}{ Faeces } & (Intercept) & -1.270 & 0.607 & $(-2.460,-0.080)$ & \\
\hline & OSR & 4.773 & 2.579 & $(1.437,9.813)$ & 1.00 \\
\hline & Fem & 5.625 & 2.137 & $(-0.282,9.827)$ & 1.00 \\
\hline & Fem $\times$ OSR & 5.085 & 3.347 & $(-1.475,11.645)$ & 0.84 \\
\hline & $\mathrm{Tl}$ & -1.435 & 2.843 & $(-7.007,4.138)$ & 0.22 \\
\hline & Ospe & 1.205 & 2.525 & $(-3.745,6.153)$ & 0.22 \\
\hline \multirow{5}{*}{ Intestines } & (Intercept) & -1.023 & 0.424 & $(-1.854,-0.192)$ & \\
\hline & OSR & 0.255 & 0.549 & $(-0.820,1.330)$ & 0.18 \\
\hline & Fem & 0.033 & 0.602 & $(-1.147,1.214)$ & 0.16 \\
\hline & $\mathrm{Tl}$ & -0.100 & 0.776 & $(-1.622,1.422)$ & 0.17 \\
\hline & Ospe & -0.673 & 1.618 & $(-3.843,2.498)$ & 0.29 \\
\hline
\end{tabular}

OSR, operational sex ratio; Fem, number of females; Fem x OSR, Fem and OSR interaction; TI, total number of helminths; Ospe, number of other non-Spauligodon helminths.

*Effect sizes have been standardized on two SD following Gelman (2008).

total number of helminths per host: $29.03 \% \pm 4.06$; number of females per host: $26.10 \% \pm 2.13)$.

\section{Discussion}

In this study, we identified the existence of male dimorphism within six different Spauligodon species, characterized by a major (MA) and minor (MI) male morphotype. Despite the considerable morphological differences between them, morphs from the same host or within the same host population were phylogenetically related (Figs 2 and 3). In a previous study, Ainsworth (1990) reported the presence of male dimorphism also in the Pharyngodonidae family, in two Skrjabinodon species (S. trimorphi and S. poicilandri) infecting lizards from New Zealand, with a similar pattern: a smaller male morph (morph 2) which was found at lower intensity levels relative to the larger male morph (morph 1). In her study, both male morphs were haploid and did not present differences in the sperm cells, indicating that both were fertile. There are several common features shared between the minor morph found in Skrjabinodon spp. and those of Spauligodon reported in this study. These include overall smaller and thinner body size, reduction or absence of lateral alae, presence of a spicule, smaller aspinose tail, a curled posterior end and low intensities, almost never exceeding one individual per host. It is important to stress here that, even if the MI morph of Spauligodon species presents morphological characteristics best fitting the taxonomic description of Skrjabinodon males, this does not necessarily mean that the genus Skrjabinodon does not exist. Actually, preliminary results have shown that Skrjabinodon poicilandri from New Zealand is a sister taxa to Spauligodon (unpublished data). From an evolutionary perspective, the maintenance of an overall similar male phenotype across different taxa may imply that these features represent ancestral character states. In this case, contrary to the intrafamilial relationships proposed by Petter \& Quentin (1976), Skrjabinodon should be considered plesiomorphic in the evolution of pharyngodonid nematodes that evolved in carnivorous hosts.

Due to the level of uncertainty in the results of ancestral state reconstruction, we cannot determine whether presence of the MI morph represents independent instances of evolution of similar traits due to convergence or parallelism, or if it constitutes a plesiomorphic trait that was lost multiple times. However, recurrent parallel forms may suggest ancestral developmental plasticity for producing both male morphs (West-Eberhard, 2005). Additionally, the nonappearance of MI in other Spauligodon species may be a lack of detection resulting from low MI prevalence and intensity values rather than a true absence. Even within those species where the MI morph has been reported, we did not find it occurring in all sampled populations (e.g. in all sampled populations of S. occidentalis from the Canary Islands). In insects, male dimorphism has repeatedly evolved, and its maintenance is assumed to be dependent on spatial and temporal heterogeneity in the environment (Schwander $\delta$ Leimar, 2011).

Although the intensity of MA was positively associated with the intensities of females in the two types of samples, no significant association was found between MI and other variables in both types of samples. In oxyurid nematodes, males are produced parthenogenetically and typically are expelled in faeces before they become infective (Adamson, 1990). Interestingly, autoinfective eggs have also been reported (Adamson, 1990). Thus, in high intensities, females may be producing autoinfective haploid eggs which develop faster than females allowing offspring to mate with their 
mother (see Adamson, 1990). The lack of agreement between the results collected from faeces and those from intestines makes it difficult to draw inferences on the factors driving the presence of MI morph and restricting their intensity per host. The operational sex ratio was found to be a good predictor for the presence of MI morph, but this was only valid for faeces and not for intestines. This discrepancy has been already discussed elsewhere (see Jorge et al., 2013), with intestines being the most reliable source of information for ecological studies of parasite associations. Therefore, relying only the intestine data, no variable was found to be a significant predictor of the presence of the MI morph. Consequently, we were not able to attribute the presence of this alternative male morph to any of the ecological variables investigated. We recognize that the present study is bound by several interpretational limitations which originate from the fact that the phenomenon is rare (only 24 samples of 593 intestinal samples and 15 of 323 faecal samples, contained MI males), thus resulting in a lack of power to detect any significant predictor. However, when the ratio between presence and absence of MI male morph was equalized in post hoc analyses, we still failed to find any variable to be a significantly important predictor of MI male morph presence. Significant effects were only detected in $1 \%$ to $14 \%$ of the repetitions, which could simply result from a random effect. This suggests that the lack of sensitivity of the model might not simply be due to the rarity of the MI male morphotype, which indirectly suggests that, even by increasing our sample, we may not be able to change the outcome of our present analyses.

We cannot completely determine the nature of male dimorphism in these elusive parasitic nematodes. Male dimorphism could also result from a genetic disorder or other environmental and/or physiological factors. However, male dimorphism is common in different Spauligodon species, presenting the same low prevalence pattern. If this was a result from a genetic disorder, why would it be so common? It may instead be a case of ART, in which both morphs are the outcome, or are linked to, different reproductive strategies and contrasting mating tactics. In this case, we would expect that in some conditions the MI male morph would perform better than the MA male morph. Is the relative fitness of the MI morph higher at lower frequencies? Without an answer to that question, another explanation is that the phenomenon is a case of alternative adaptation, allowing the two male morphs to occupy more than one sympatric niche and thereby increasing the adaptive potential of the species, and having nothing to do with reproductive tactic. But why would the MI male morph be only present in such low numbers (only once exceeding one per host) if it occupies a niche different from that of the MA morph? To date, no developmental, physiological or behavioural experiments have been performed to test any of these possibilities.
However, we tend to favour the hypothesis of ART for the following reasons: (i) physiologically, in both male morphs a brownish secretion located near the genital area was found, similar to that observed when MA morphs copulate with females (unpublished data), suggesting that the MI morph are also fertile. This is additionally supported with evidence from Ainsworth (1990) regarding fertility of Skrjabinodon male morphs, that is, both male morphs were haploid and did not present differences in the sperm cells, indicating that both were fertile. Fertility of the MI morph is a necessary condition for the ART hypothesis to be accepted. And (ii) ecologically, even if we did not find a good predictor for the presence of the MI morph, including male competition and overall helminths intensity, the characteristics of the MI (i.e. smaller size, reduced characters, lower intensity values in the host) resemble those reported in several examples of ART (see Oliveira et al., 2008). Regardless of its evolutionary origin, the presence of two morphs is generally interpreted in terms of difference in fitness. The lower prevalence and intensity of the MI morph suggest a lower fitness or, alternatively, that the tactic associated with the MI morph is only more successful when rare. In the latter scenario, the MI morph may be under a strong negative frequency-dependent selection, as observed in damselflies (Iserbyt et al., 2013), such that it is maintained at very low intensity levels. However, the presence of three MI individuals in a faecal sample is indicative that in some conditions this may be overcome. Then again, it is still unclear which are the fitness trade-offs of the two different male morphotypes and particularly how the MI morph is maintained in the population or what is its adaptive potential.

Understanding the evolution of alternative phenotypes and assessing how selection acts in decision-making processes (Brockmann, 2001; Oliveira et al., 2008) requires an integrative approach including developmental, physiological, morphological and behavioural studies. We admit that most questions regarding the origin of the two male morphotypes can only be answered by means of experimental procedures. However, given the parasitic nature and frequency of this system, and especially the lack of knowledge on physiology and development, the underlying mechanisms maintaining the MI morph remain unknown. Nevertheless, our findings will need to be replicated in the future on a taxonomically and numerically enlarged data set. Unfortunately, such additional sampling is currently not feasible due to the elusive nature of these nematodes; in particular, to increase our chances of detecting such a rare event, we would need to carry out invasive sampling (i.e. sacrifice the host). The presence of different morphotypes highlights once more the importance of integrating not only morphological characters into the analyses, but also other data sources, particularly genetic data. This is especially important for taxonomic groups for which 
investigating behaviour and physiology is intrinsically difficult and consequently groups that are characterized solely on morphological traits. If the understanding of alternative phenotypes is still far from complete in some well-studied groups (see Oliveira et al., 2008), the study of male dimorphism in parasites clearly lags much further behind.

\section{Acknowledgments}

FJ was funded through a Doctoral grant (SFRH/BD/ 77332/2011) and AP with an IF FCT contract (IF/01257/ 2012). This research is part of the projects 'Genomics and Evolutionary Biology' cofinanced by North Portugal Regional Operational Programme 2007/2013 (ON.2 - O Novo Norte), under the National Strategic Reference Framework (NSRF), through the European Regional Development Fund (ERDF), PTDC/BIA-BEC/101256/ 2008 of FCT (Portugal), FCOMP-01-0124-FEDER007062 COMPETE program and 'Preserving Armenian biodiversity: Joint Portuguese - Armenian program for training in modern conservation biology' of Gulbenkian Fundation (Portugal). We thank Cabildos Insulares (Island Authorities) from Lanzarote, Fuerteventura, Gran Canaria, Tenerife, La Palma, La Gomera and El Hierro from Spain; the ICNB from Portugal, Turkish Environmental Authority (project 2009.KB.FEN.003 by Fauna and Flora Research and Application Center, Dokuz Eylul University) and licence no. 07/2008 by Direcção Geral do Ambiente, MAA, Cape Verdean Government for research permits. Thanks to the members of CIBIO and all the collaborators who helped in the collection of samples. Special thanks to K. Lange, P. Tarroso and A. Kaliontzopoulou for their helpful comments.

\section{References}

Adamson, M.L. 1990. Haplodiploidy in the Oxyurida: decoupling the evolutionary processes of adaptation and speciation. Ann. Parasitol. Hum. Comp. 65 (Suppl 1): 31-35.

Ainsworth, R. 1990. Male dimorphism in two new species of nematode (Pharyngodonidae, Oxyurida) from New Zealand lizards. J. Parasitol. 76: 812-822.

Bartoń, K. 2009. MuMIn: multi-model inference. R package, version 1.9.5. Available at: http://r-forge.r-project.org/ projects/ mumin/.

Bastiaans, E., Morinaga, G., Castañeda Gaytán, J.G., Marshall, J.C. \& Sinervo, B. 2013. Male aggression varies with throat color in 2 distinct populations of the mesquite lizard. Behav. Ecol. 24: 968-981.

Biomatters 2013. Geneious v.6.1.2 2013. Available at: http:// www.geneious.com/.

Brockmann, H.J. 2001. The evolution of alternative strategies and tactics. Adv. Study Behavior. 30: 1-51.

Burnham, K.P. \& Anderson, D.R. 2002. Model Selection and Multimodel Inference: A Practical Information-Theoretic Approach, 2nd edn. Springer, Berlin.
Buzatto, B.A., Requena, G.S., Lourenço, R.S., Munguía-Steyer, R. \& Machado, G. 2011. Conditional male dimorphism and alternative reproductive tactics in a Neotropical arachnid (Opiliones). Evol. Ecol. 25: 331-349.

Calsbeek, B., Hasselquist, D. \& Clobert, J. 2010. Multivariate phenotypes and the potential for alternative phenotypic optima in wall lizard (Podarcis muralis) ventral colour morphs. J. Evol. Biol. 23: 1138-1147.

Cogliati, K.M., Neff, B.D. \& Balshine, S. 2013. High degree of paternity loss in a species with alternative reproductive tactics. Behav. Ecol. Sociobiol. 67: 399-408.

Craig, B.H., Pilkington, J.G. \& Pemberton, J.M. 2010. Sex ratio and morphological polymorphism in an isolated, endemic Teladorsagia circumcincta population. J. Helminthol. 84: 208215.

Cunningham, C.W., Omland, K.E. \& Oakley, T.H. 1998. Reconstructing ancestral character states: a critical reappraisal. Trends Ecol. Evol. 13: 361-366.

Darriba, D., Taboada, G.L., Doallo, R. \& Posada, D. 2012. jModelTest 2: more models, new heuristics and parallel computing. Nat. Methods 9: 772.

Falk, B.G. \& Perkins, S.L. 2013. Host specificity shapes population structure of pinworm parasites in Caribbean reptiles. Mol. Ecol. 22: 4576-4590.

Felsenstein, J. 1985. Confidence-limits on phylogenies-An approach using the bootstrap. Evolution 39: 783-791.

Floyd, R.M., Rogers, A.D., Lambshead, J.D. \& Smith, C.R. 2005. Nematode-specific PCR primers for the 18S small subunit rRNA gene. Mol. Ecol. Notes 5: 611-612.

Gelman, A. 2008. Scaling regression inputs by dividing by two standard deviations. Stat. Med. 27: 2865-2873.

Gelman, A., Su, Y.-S., Yajima, M., Hill, J., Pittau, M.G., Kerman, J. et al. 2009. Arm: data analysis using regression and multilevel / hierarchical models. R package, version 1.6-09. Available at: http://CRAN.R-project.org/package $=$ arm .

Grillo, V., Craig, B.H., Wimmer, B. \& Gilleard, J.S. 2008. Microsatellite genotyping supports the hypothesis that Teladorsagia davtiani and Teladorsagia trifurcata are morphotypes of Teladorsagia circumcincta. Mol. Biochem. Parasitol. 159: 59-63.

Gross, M.R. 1996. Alternative reproductive strategies and tactics: diversity within sexes. Trends Ecol. Evol. 11: 92-98.

Grueber, C.E., Nakagawa, S., Laws, R.J. \& Jamieson, I.G. 2011. Multimodel inference in ecology and evolution: challenges and solutions. J. Evol. Biol. 24: 699-711.

Guindon, S. \& Gascuel, O. 2003. A simple, fast and accurate method to estimate large phylogenies by maximum-likelihood. Syst. Biol. 52: 696-704.

Harrell, F.E., with contributions from Dupont, C. and many others. 2013. Hmisc: Harrell Miscellaneous. R package version 3.10-1.1. Available at: http://CRAN.R-project.org/package $=$ Hmisc.

Hoberg, E.P. \& Abrams, A. 2001. Synlophe in Ostertagia cf. kasakhstanica (Nematoda: Ostertagiinae), the minor morphotype of 0 . bisonis from western North America. J. Parasitol. 87: 1181-1184.

Hoberg, E.P., Abrams, A., Pilitt, P.A. \& Kutz, S.J. 2012. Discovery and description of the 'davtiani' morphotype for Teladorsagia boreoarcticus (Trichostrongyloidea: Ostertagiinae) abomasal parasites in muskoxen, Ovibos moschatus, and caribou, Rangifer tarandus, from the North American Arctic: 
implications for parasite faunal diversity. J. Parasitol. 98 355-364.

Hornero, M.J. \& Roca, V. 1992. Helmintofauna de Podarcis lilfordi (Günther, 1874) (Sauria, Lacertidae) de los islotes de Menorca (Islas Baleares, Mediterráneo Occidental). Misc. Zool. 16: 1-6.

Horton, B.M., Hauber, M.E. \& Maney, D.L. 2012. Morph matters: aggression bias in a polymorphic sparrow. PLOS ONE 7: e48705.

Huelsenbeck, J.P. \& Ronquist, F. 2001. MRBAYES: Bayesian inference of phylogeny. Bioinformatics 17: 754-755.

Iserbyt, A., Bots, J., Gossum, H.V. \& Sherratt, T.N. 2013. Negative frequency-dependent selection or alternative reproductive tactics: maintenance of female polymorphism in natural populations. BMC Evol. Biol. 13: 139.

Jorge, F., Roca, V., Perera, A., Harris, D.J. \& Carretero, M.A. 2011. A phylogenetic assessment of the colonisation patterns in Spauligodon atlanticus Astasio-Arbiza et al., 1987 (Nematoda: Oxyurida: Pharyngodonidae), a parasite of lizards of the genus Gallotia Boulenger: no simple answers. Syst. Parasitol. 80: 53-66.

Jorge, F., Carretero, M.A., Perera, A., Harris, D.J. \& Roca, V. 2012. A new species of Spauligodon (Nematoda: Oxyurida: Pharyngodonidae) in geckos from São Nicolau island (Cape Verde) and its phylogenetic assessment. J. Parasitol. 98: 160166.

Jorge, F., Carretero, M.A., Roca, V., Poulin, R. \& Perera, A. 2013. What you get is what they have? Detectability of intestinal parasites in reptiles using faeces. Parasitol. Res. 112: 4001-4007.

Katoh, K., Misawa, K., Kumar, K. \& Miyata, T. 2002. MAFFT: a novel method for rapid multiple sequence alignment based on fast Fourier transform. Nucleic Acids Res. 30: 3059-3066.

Kvarnemo, C. \& Ahnesjö, I. 1996. The dynamics of operational sex ratios and competition for mates. Trends Ecol. Evol. 11: 404-408.

Maddison, W.P. \& Maddison, D.R. 2011. Mesquite: a modular system for evolutionary analysis. Version 2.75. Available at: http://mesquiteproject.org.

Mank, J.E. \& Avise, J.C. 2006. The evolution of reproductive and genomic diversity in ray-finned fishes: insights from phylogeny and comparative analysis. J. Fish Biol. 69: 1-27.

Messing, J. 1993. M13 cloning vehicles: their contribution to DNA sequencing. Methods Mol. Biol. 23: 9-22.

Nakagawa, S. \& Freckleton, R.P. 2010. Model averaging, missing data and multiple imputation: a case study for behavioural ecology. Behav. Ecol. Sociobiol. 65: 103-116.

Neff, B.D. \& Svensson, E.I. 2013. Polyandry and alternative mating tactics. Phil. Trans. R. Soc. B 368: 20120045.

Oliveira, R.F., Taborsky, M. \& Brockmann, H.J. 2008. Alternative Reproductive Tactics. Cambridge University Press, Cambridge.

Petter, A.J. \& Quentin, J.C. 1976. Keys to genera of the Oxyuroidea. In: Keys to the Nematode Parasites of Vertebrates (R.C. Anderson, A.G. Chabaud \& S. Willmott, eds), pp. 1-30. C.I.H. CAB International, London.
Pizzatto, L. \& Dubey, S. 2012. Colour-polymorphic snake species are older. Biol. J. Linn. Soc. 107: 210-218.

Prosser, S.W., Velarde-Aguilar, M.G., León-Règagnon, V. \& Hebert, P.D. 2013. Advancing nematode barcoding: a primer cocktail for the cytochrome c oxidase subunit I gene from vertebrate parasitic nematodes. Mol. Ecol. Resour. 13: 11081115.

R Core Team 2013. R: A Language and Environment for Statistical Computing. R Foundation for Statistical Computing, Vienna, Austria. Available at: http://www.R-project.org/.

Roca, V., López-Balaguer, E. \& Hornero, M.J. 1989. Helmintofauna de Podarcis hispanica (Steindachner, 1870) y Podarcis bocagei (Seoane, 1884) (Reptilia: Lacertidae) en el Cuadrante Noroccidental de la Península Ibérica. Rev. Iber. Parasitol. 49: 127-135.

Roca, V., Carretero, M.A., Llorente, G.A., Montori, A. \& Martin, J.E. 2005. Helminth communities of two lizard populations (Lacertidae) from Canary Islands (Spain). Host diet-parasite relationships. Amphibia-Reptilia 26: 535-542.

Schwander, T. \& Leimar, O. 2011. Genes as leaders and followers in evolution. Trends Ecol. Evol. 26: 143-151.

Shuster, S.M. \& Wade, M.J. 2003. Mating Systems and Strategies. Princeton University Press, Princeton, New Jersey.

Taborsky, M., Oliveira, R.F. \& Brockmann, H.J. 2008. The evolution of alternative reproductive tactics: concepts and questions. In: Alternative Reproductive Tactics: An Integrative Approach (R.F. Oliveira, M. Taborsky \& H.J. Brockmann, eds), pp. 1-21. Cambridge University Press, Cambridge.

Tamura, K., Peterson, D., Peterson, N., Stecher, G., Nei, M. \& Kumar, S. 2011. MEGA5: molecular evolutionary genetics analysis using maximum likelihood, evolutionary distance, and maximum parsimony methods. Mol. Biol. Evol. 28: 2731-2739.

Tomkins, J.L. \& Hazel, W. 2007. The status of the conditional evolutionarily stable strategy. Trends Ecol. Evol. 22: 522-528.

West-Eberhard, M.J. 1986. Alternative adaptations, speciation, and phylogeny (a review). Proc. Natl. Acad. Sci. USA 83: 1388-1392.

West-Eberhard, M.J. 2005. Developmental plasticity and the origin of species differences. Proc. Natl. Acad. Sci. USA 102: 6543-6549.

Whiting, M.F. 2002. Mecoptera is paraphyletic: multiple genes and phylogeny of Mecoptera and Siphonaptera. Zool. Scr. 31: 93-104.

\section{Supporting information}

Additional Supporting Information may be found in the online version of this article:

Table S1 Number of samples collected in this study from each host species. (I: intestine; F: faeces).

\section{Data deposited at Dryad: doi:10.5061/dryad.cb5f3}

Received 21 January 2014; accepted 16 April 2014 